\title{
Pedicled local flaps: a reliable reconstructive tool for partial breast defects
}

\author{
Sherif Youssif ${ }^{1,2}$, Youssef Hassan ${ }^{2}$, Ahmed Tohamy ${ }^{2}$, Samir Eid ${ }^{3}$, Tarek Ashour ${ }^{1,4}$, Marco Malahias ${ }^{1}$, \\ Haitham Khalil $^{1}$
}

${ }^{1}$ Plastic and Reconstructive Surgery Division, Good Hope Hospital, University Hospitals Birmingham NHS Trust, Birmingham, UK; ${ }^{2}$ Plastic Surgery Department, Faculty of Medicine, Assiut University Hospitals, Assiut, Egypt; ${ }^{3}$ Department of Clinical Oncology and Nuclear Medicine, Faculty of Medicine, Assiut University, Assiut, Egypt; ${ }^{4}$ Plastic Surgery Department, Cairo University Hospitals, Cairo, Egypt

Contributions: (I) Conception and design: S Youssif, H Khalil; (II) Administrative support: None; (III) Provision of study materials or patients: None; (IV) Collection and assembly of data: S Youssif, H Khalil; (V) Data analysis and interpretation: All authors; (VI) Manuscript writing: All authors; (VII) Final approval of manuscript: All authors.

Correspondence to: Mr Sherif Youssif, MSc. Plastic Surgery Fellow, Plastic and Reconstructive Surgery Division, Good Hope Hospital, University Hospitals Birmingham NHS Trust, Rectory Road, Sutton Coldfield, Birmingham, B75 7RR, West Midlands, UK. Email: sherifyoussif123@gmail.com; Mr Haitham Khalil, MSc, MRCS(Ed), MD, FRCS(Eng). Consultant Oncoplasty and Reconstructive Surgeon, Plastic and Reconstructive Surgery Division, Good Hope Hospital, University Hospitals Birmingham NHS Trust, Sutton Coldfield, Birmingham, B75 7RR, West Midlands, UK. Email: haitham.khalil@heartofengland.nhs.uk.

Background: Breast conserving surgery (BCS) followed by radiotherapy has gained great popularity in the treatment of breast cancer over the past years. However, radiation therapy can lead to many unfavourable aesthetic outcomes including significant volume/skin deficiency, nipple areola complex distortion and skin contraction. We present our experience in using pedicled perforator flaps to tackle the resultant partial breast defects or deformities.

Methods: A retrospective data analysis study on Thirty patients with post breast conserving surgery (BCS) partial breast defects who were managed with pedicled per-forator flaps including muscle sparing latissimus dorsi muscle flap (MSLD), thoraco-dorsal artery perforator flap (TDAP) and intercostal artery perforator flap (ICAP) in the period between December 2008 and December 2018.

Results: Defects were in all quadrants apart from the upper inner quadrant. The reconstructive techniques included TDAP flap 6/30 (20\%), MSLD flap 20/30 (66.7\%), AICAP flap 4/30 (13.3\%). Age ranges 22-35 (mean 29). All flaps showed complete survival, one nipple areola complex superficial epidermolysis was experienced, and one patient presented with fat necrosis. No resultant donor site morbidity apart from scar revision for excess skin at the axillary fold in one patient. The overall satisfaction reached $94 \%$ with only 8 patients who required lipofilling to maximize the cosmetic outcome.

Conclusions: The availability of a range of reliable techniques including thoracodorsal/intercostal artery perforator flap (TAP/ICAP) and muscle sparing lattissimus dorsi flap (MSLD) allow optimum results to be achieved in the treatment of partial breast defects following breast conserving surgery.

Keywords: Breast cancer; breast conserving surgery; partial breast reconstruction; perforator flap

Submitted May 17, 2019. Accepted for publication Aug 28, 2019.

doi: 10.21037 /gs.2019.09.06

View this article at: http://dx.doi.org/10.21037/gs.2019.09.06 


\section{Introduction}

Over time, the advances in adjuvant therapy in the management of breast cancer patients has led to less radical surgical approach and more women became candidates for breast conservation. However, the resulting significant volume and skin deficiency, nipple areola complex distortion and skin contraction can lead to unsatisfactory aesthetic outcomes (1). As a result, oncoplastic surgical techniques were developed and subsequently became invaluable to the field of reconstructive surgery, as well as to the comprehensive treatment of breast cancer. Oncoplastic techniques minimize breast deformities following cancer resection and treatment while maintaining equivalent oncologic outcomes (2). These techniques include local tissue rearrangement, local-regional flap transfer, fat grafting and reduction mammoplasty (3). Depending on the amount of gland resection and the size of the breast, these different techniques can be applied. When the defect is around 30\% of the breast volume, pedicled perforator flaps should be the method of choice in partial breast reconstruction (4). What characterizes perforator flaps is sparing of the underlying muscle or the functional motor nerves and less donor-site morbidity. The pedicled perforator flaps commonly used for breast reconstruction are the Muscle-sparing Latissimus Dorsi (MS-LD), the TDAP flap, the intercostal artery perforator (ICAP) flap, and the serratus anterior artery perforator (SAAP) flap (5). These flaps can be used either for partial breast reconstruction after breast conserving surgery to add more volume for previously reconstructed breast or for total breast reconstruction with expander or implant (6).

\section{Methods}

This study was conducted on 30 patients who were presented to plastic surgery department, hope hospital, university hospitals Birmingham NHS Foundation trust, UK. These patients underwent autologous loco-regional perforator flaps for management of partial breast defects post conservative surgery of breast cancer by senior author (HK) in the period between December 2008 and December 2018. The aim of this study is to assess the reliability of utilizing pedicled autologous tissue transfer including muscle sparing latissimus dorsi flap (MSLD), thoracodorsal artery perforator flap (TAP) and intercostal artery perforator flap (ICAP) flap in management of partial breast defects following breast conserving surgery. Patients included in this study were those who developed partial breast defects (lateral, medial, upper poles, lower poles and central) after breast conserving surgery (BCS) and whom have completed their oncological treatment.

\section{Selection criteria}

All patients were managed through a multidisciplinary breast oncoplastic team to optimize their peri-operative treatment plan. Preoperative preparation included clinical assessment to determine the extent of the defect, degree of the volume and skin deficiency, radiological investigation including mammogram and CT scan staging for patient with high risk for metastatic potential. The indications to consider partial breast defect surgical treatment included patients with over $20 \%$ of volume deficiency with or without skin deficiency or retraction and nipple areola complex distortion in the lateral quadrants and the lower pole of the breast. Partial breast defects in the upper inner quadrant were excluded from this cohort as it was deemed anatomically difficult to recruit local perforator flaps to this zone without causing distortion of the breast and because of the long distance from the donor site to the recipient zone. Other factors were taken in consideration during planning including previous local radiotherapy and surgery and/or associated general risk factors as diabetes, active smoking, chemotherapy, morbid obesity and planning for contralateral breast symmetrisation procedure.

\section{Data collection}

A prospectively maintained data base was used to identify the patients and their records were assessed retrospectively. The demographic information and potential identifiable risks and co comorbidities, previous history of malignancy and treatment were collected. Tumour characteristics and details of adjuvant therapy including either chemotherapy or radiotherapy were also documented. The results of the radiological diagnostic workup included mammogram and CT scan staging were noted. The operative details identified the site and size of the defect post release of the surgical scar. In addition, the methods of soft tissue coverage (local flap) and whether contralateral breast symmetrisation was performed were also documented. The postoperative outcomes in terms of length of stay, complications particularly surgical site infection (SSI) and wound dehiscence, flap survival, revisional surgery, symmetry, donor site morbidity and patient satisfaction were identified 

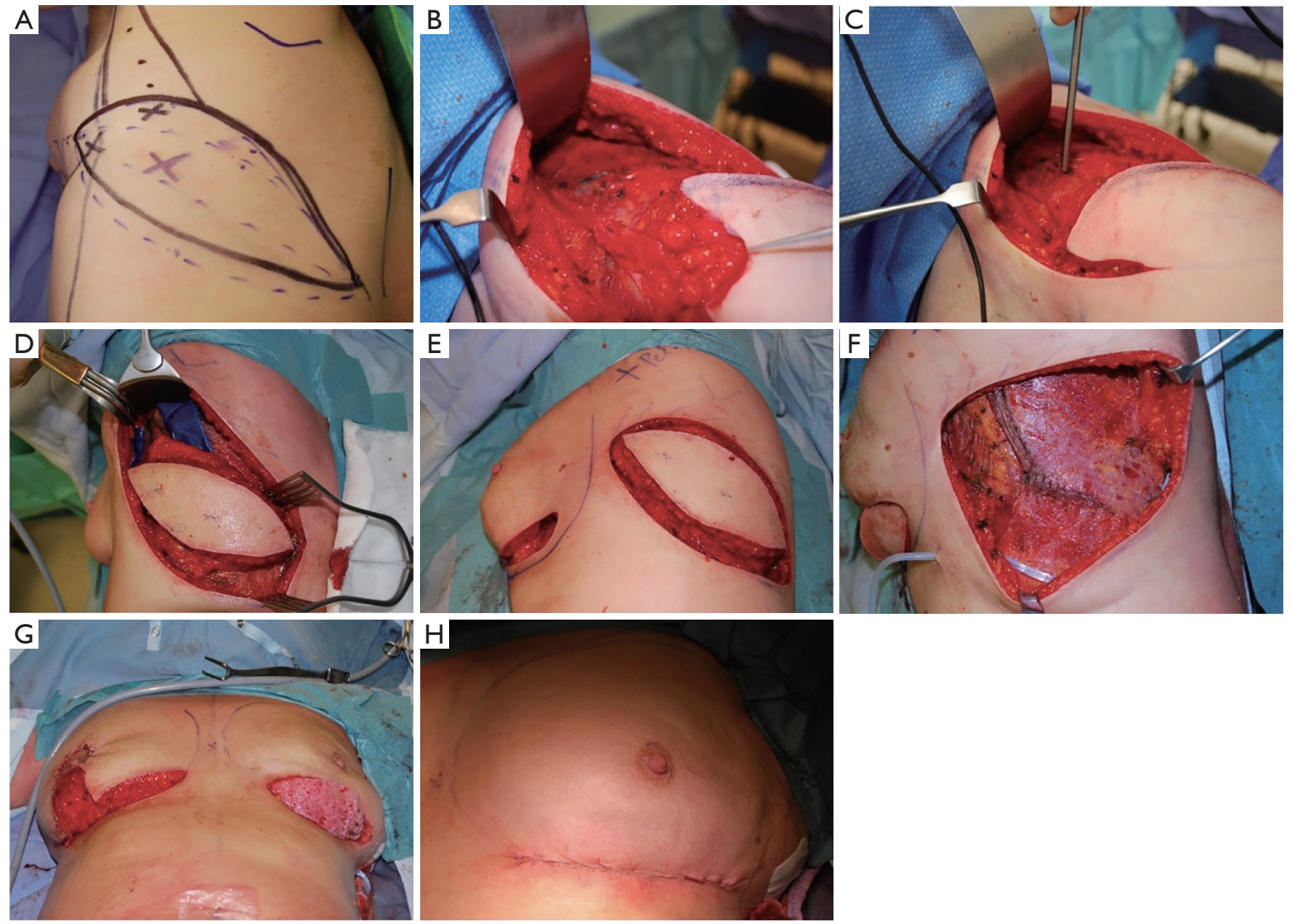

Figure 1 Operative details of harvesting muscle sparing latissimus dorsi muscle flap for reconstruction of partial breast defects. (A) Preoperative markings of muscle sparing latissimus flap and identification of the perforators with hand held Doppler; (B) intraoperative photograph showing dissection of the back skin from the LD muscle and identification of the anterior muscle border; (C) identification of the flap pedicle using intraoperative Doppler; (D) splitting of the LD muscle creating 4-5 cm muscle strip that involves the flap pedicle; (E) simultaneous preparation of the recipient site and release of all scar tissue down to the pectoralis fascia; (F) direct closure of the muscle edges after tunneling the flap to the recipient site to obtain proper alignment of the muscle fibers and stop any bleeding from muscle edges; $(\mathrm{G}, \mathrm{H})$ repositioning of the patient in supine position and deepthelialization of the flap skin before burying it and direct closure of overlying breast skin.

from the patient's records. Finally, patients were asked to fill out a study-specific, standardized questionnaire for overall satisfaction during regular follow up clinics.

\section{Operative consideration}

Depending on the site of the defect the local flap was designed either (TAP/MSLD/or ICAP flap). The skin flap and the anterior border of the LD muscle are marked preoperatively with the patient in the standing position.
A pinch test of the transverse skin crease below the axillary fat pad is used to identify the widest portion of the transversely oriented flap. This crease is continuous with the inframammary fold. The patient is positioned intraoperatively in the lateral decubitus position and recapping on flap markings is done. Irrespective of the flap choice preoperative hand held Doppler identification of the perforators was performed followed by designing the flap skin island in the breast strap line (Figure 1A). This is followed by standard meticulous dissection the flap 


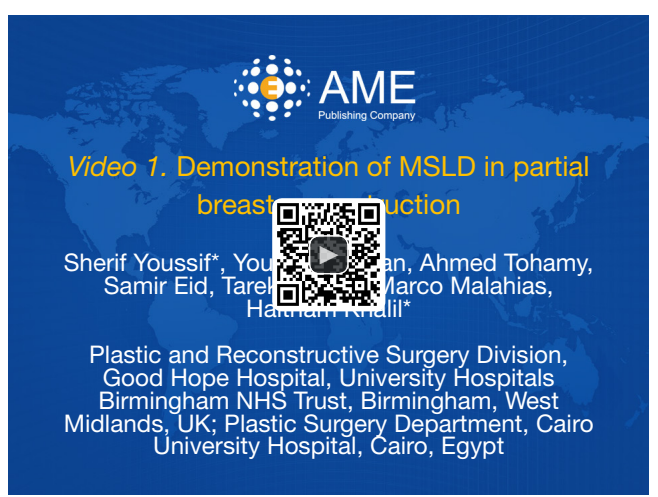

Figure 2 Demonstration of MSLD in partial breast reconstruction (7). Pre-operative markings are done before patient is positioned intraoperatively in the lateral decubitus position and hand held Doppler identification of the perforators was performed. This is followed by standard meticulous dissection the flap and isolation of the pedicle. The latissimus muscle is then divided distal to the flap to create a $4-5 \mathrm{~cm}$-wide muscle strip, and a retrograde dissection of the flap and overlying soft tissue paddle is performed. Simultaneously, the recipient site is prepared, and the flap is tunneled to the receipt defect. Finally, the back incision is closed in layers over a closed-suction drain. The patient is then repositioned into the supine position on the operating room table with the arm abducted and supported, and in some cases deepithilization of the excess cutaneous component of the flap was performed to add to the volume of the reconstruction before burying the flap and skin closure.

Available online: http://www.asvide.com/watch/32992

and isolation of the pedicle. First, an elliptical incision is made, beginning at the inframammary fold and extending towards the midline of the back, along the inferior margin of the skin flap. The native back skin is elevated from the latissimus dorsi (LD) muscle inferiorly, and the anterior muscle border is identified (Figure 1B). The latissimus muscle is then divided distal to the flap to create a 4-5 cm-wide muscle strip, and a retrograde dissection of the flap and overlying soft tissue paddle is performed. The anterior descending branch of the thoracodorsal artery is identified with an intra-operative Doppler probe, and muscle splitting is then further performed (Figure 1C,D). In some cases, the transverse branch was ligated to add more length to the TD pedicle. Simultaneously, the recipient site is prepared, and the defect is created post excision and release of all the scar tissue down to the pectoralis facia which is sent for histopathological examination (Figure 1E). In addition, rigotomies may be required in the surrounding breast tissue to allow further expansion of the breast skin envelope. Direct closure of the muscle edges is performed to obtain proper alignment of muscle fibers and stop any bleeding from muscle edges (Figure $1 F$ ). Once the flap is harvested, tunneling and insetting of the flap to the receipt defect followed by shaping and securing of the flap into the defect was performed. Finally, the back incision is closed in layers over a closed-suction drain. The patient is then repositioned into the supine position on the operating room table with the arm abducted and supported, and in some cases deepithilization of the excess cutaneous component of the flap was performed to add to the volume of the reconstruction before burying the flap and skin closure (Figure 1G,H). Fine tuning of the insetting of the flap is always performed while the patient is in the sitting position to achieve the best aesthetic outcome (Figure 2). Additional volume was achieved in four patients through complete subglandular dissection to accommodate an implant with immediate contralateral symmetrisation breast augmentation. The subglandular plane was adopted in three cases and subpectoral plane for one case. On the other hand; eight patients required second stage lipofilling to enhance the aesthetic outcomes. Suction drains were inserted deep to the flap and subcutaneous tissue. Prior to standard skin closure intercostal and pectoral nerve block was performed. Standard flap observation chart protocol was followed, including Doppler signal, skin colour, temperature and capillary refilling. All patients received antibiotics intravenous therapy on induction of anesthesia, followed by oral therapy for the duration of time the drains remained in situ. All patients were immediately put in supportive brassier.

\section{Results}

Between 2008-2018, 30 patients presented with partial breast defects post wide local excision and radiotherapy for breast cancer with an age range of 23-61 years (mean 45) were treated. Their body mass index (BMI) ranged from 22-35 (mean 29). The duration from the initial breast cancer diagnosis and treatment to their presentation for correction of the partial breast defect ranged from 12-60 months (mean 28). All cases were presented with unilateral partial breast defect post Breast conserving surgery. The percentage of the deficient breast volume to the total breast volume was clinically assessed and ranged from $20-40 \%$ (mean $27 \%$ ). All patients had preoperative bilateral mammograms while 11 patients had CT staging 


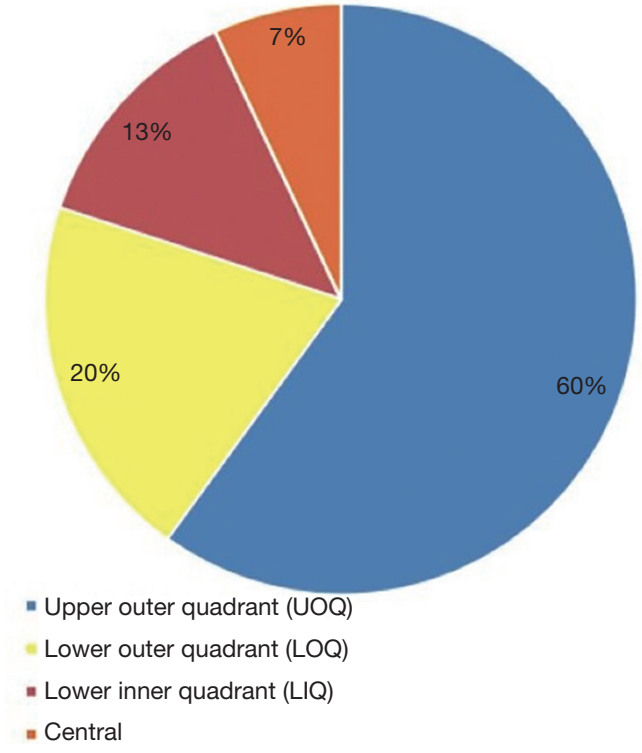

Figure 3 Distribution of reconstructed breast quadrants.

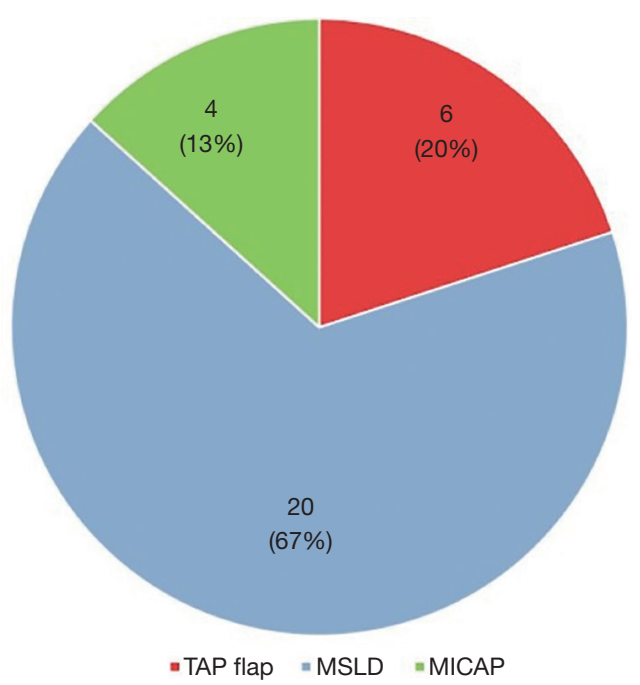

Figure 4 Type of flap used in reconstruction.

due the metastatic potential of the original pathology including high grade, vascular invasion and involved lymph nodes. All patients were non-smokers or ex-smokers which were confirmed using urine cotinine test at the time of their commencement of their treatment. The deformities were encountered in all quadrants apart from the upper inner quadrant with the upper outer quadrant (UOQ) representing the commonest site in $18 / 30(60 \%)$, lower outer quadrant (LOQ) 6/30 (20\%), lower inner quadrant (LIQ) $4 / 30(13 \%)$ and central 2/30 (7\%) (Figure 3). The
Table 1 Flap related complications

\begin{tabular}{lc}
\hline Flap related complications & No. flaps (\%) \\
\hline No complications & $28(93.3 \%)$ \\
Infection & Nil \\
Seroma & Nil \\
Haematoma & Nil \\
Total or partial flap loss & Nil \\
NAC partial necrosis & $1(3.3 \%)$ \\
fat necrosis & $1(3.3 \%)$ \\
Total complications & $2(6.6 \%)$ \\
\hline
\end{tabular}

NAC, nipple areolar complex.

reconstructive techniques included thoracodorsal artery perforator flap (TAP) 6/30 (20\%) muscle sparing latissimus dorsi flap (MSLD) 20/30 (67\%), anterior intercostal artery perforator flap (AICAP) 4/30 (13\%) (Figure 4). The maximum width of the flap skin island was $9 \mathrm{~cm}$ while the maximum length was $21 \mathrm{~cm}$. The length of hospital stay ranged from 2-4 days (mean 2.6). Additional second stage lipofilling was required in eight patients (26.7\%) to fine tune the aesthetic outcome between 6-18 months postoperatively while 4 (13.3\%) had concomitant implantbased reconstruction in both breasts. The follow-up period ranged from 9 to 52 months (mean 24.8), and all patients were evaluated by the operating surgeon. All flaps showed complete survival, one nipple areola complex superficial epidermolysis was experienced, and one patient presented with fat necrosis (Table 1). No donor site morbidity was experienced in any of the patients apart from scar revision for excess skin at the axillary fold in one patient. In all our patients, the donor site scar was aesthetically placed in the bra strap line (Figure 5). There was no local recurrence or distant metastases encountered during the follow-up period. Also, it's worth mentioning that there was no evidence of any capsular contracture in patients who had breast implants as a second stage procedure for correction of any residual deformity. The aesthetic outcome was assessed through a patient questionnaire survey during regular follow up clinics at 3, 6 and 12 months with regards to volume shape and overall satisfaction. Most of our patients had satisfactory results with reasonable breast symmetry (Figure 6). The overall satisfaction reached $94 \%$ with only 8 patients (26.7\%) who required 2 nd stage lipofilling to maximize the cosmetic outcome (Figure 7). 

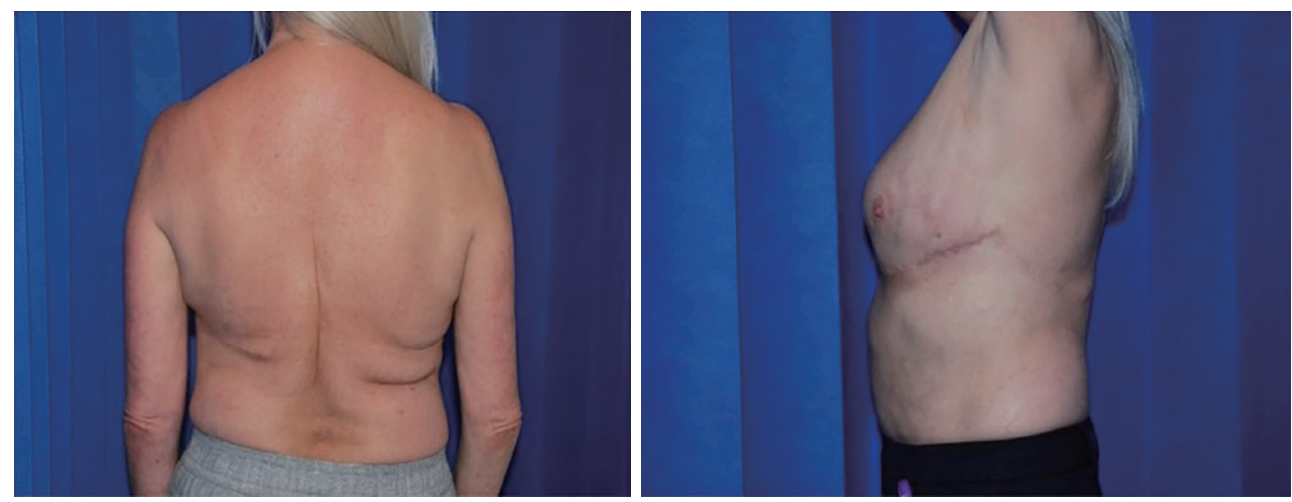

Figure 5 Post-operative photos showing the donor site scar aesthetically placed in the bra strap line.

\section{Discussion}

Techniques for partial breast reconstruction after breast conserving surgery using pedicled flaps have evolved over time, from the use of the entire LD muscle to the thoracodorsal artery perforator flap to the most recent pedicled descending branch MSLD flap which has led to a more aesthetic outcome with minimal complications (8). The full LD flap has been widely used because of advantages such as reliable vascularity, proximity to the defect and relative simplicity in dissection. Despite all its advantages, seroma and limitation of shoulder movement are very troublesome complications, making it difficult for reconstructive surgeons to choose this procedure as a primary option (9). This has led the reconstructive surgeons to start searching for a reliable option with less complications. Angrigiani was the first to introduce the concept of raising the cutaneous portion of the latissimus dorsi flap without the muscle in 1995, while the use of a pedicled thoraco-dorsal artery perforator flap in breast reconstruction was first reported in $2004(10,11)$.

Worldwide, pedicled perforator flaps has now became one of the first choices in partial breast reconstruction, resulting in an improved functional and aesthetic outcome for the patient. The ideal candidates for this reconstructive tool are patients who have a fat-skin excess at the lateral axillary region (12). We report our experience using the pedicled perforator flaps (MSLD/TDAP/ICAP) flap for management of partial breast defects in a series of 30 patients (30 flaps). Despite the presence of other alternative options as lipofilling, remodelling glanduloplasty and free flaps, we consider pedicled perforator flaps a preferable option to tackle this problem as it entails recruitment of a robust well vascularized tissue. It is reported that if local tissue rearrangement and remodelling glanduloplasty are performed as delayed reconstruction after breast-conserving surgery, patients might develop poor aesthetic outcome with high possibility of complications due to the poorquality tissues within the radiation field (13). Moreover, free abdominal tissue transfer is not a popular option in reconstruction of partial breast defects as it is a major complex procedure, needs special expertise, associated with higher donor site morbidity and considered relatively large sized flaps for these small defects. However, it might be an option for complex upper inner quadrant defects which is difficult to reconstruct with the local flaps used in our study (14). It is reported that internal mammary artery perforator flap is a reliable flap in reconstructing defects in medial half but with donor site problems. However, it was not involved in our study (15). In our hands, lipofilling and breast prothesis are considered unfavourable options in these patients because of the associated post radiotherapy complications as fat necrosis, capsular contracture and wound healing problems.

It is clear from our figures that our preference was towards the MSLD flap as 67\% (20/30) of our cases were managed using this flap. This is because the MSLD flap is relatively more robust, reliable and easier in harvesting when compared to the TDAP flap. On the other hand, the TDAP flap was reserved as a second option when the MSLD flap is an over indication as in small outer half partial breast defects. The ICAP flap was used in lower medial defects which couldn't be reached by the MSLD flap. The width of the muscle strip included in the MSLD flap in our technique ranged from $4-5 \mathrm{~cm}$. This allowed us to reliably include the anterior descending branch of the thoracodorsal artery and its muscular perforators. Also, by 

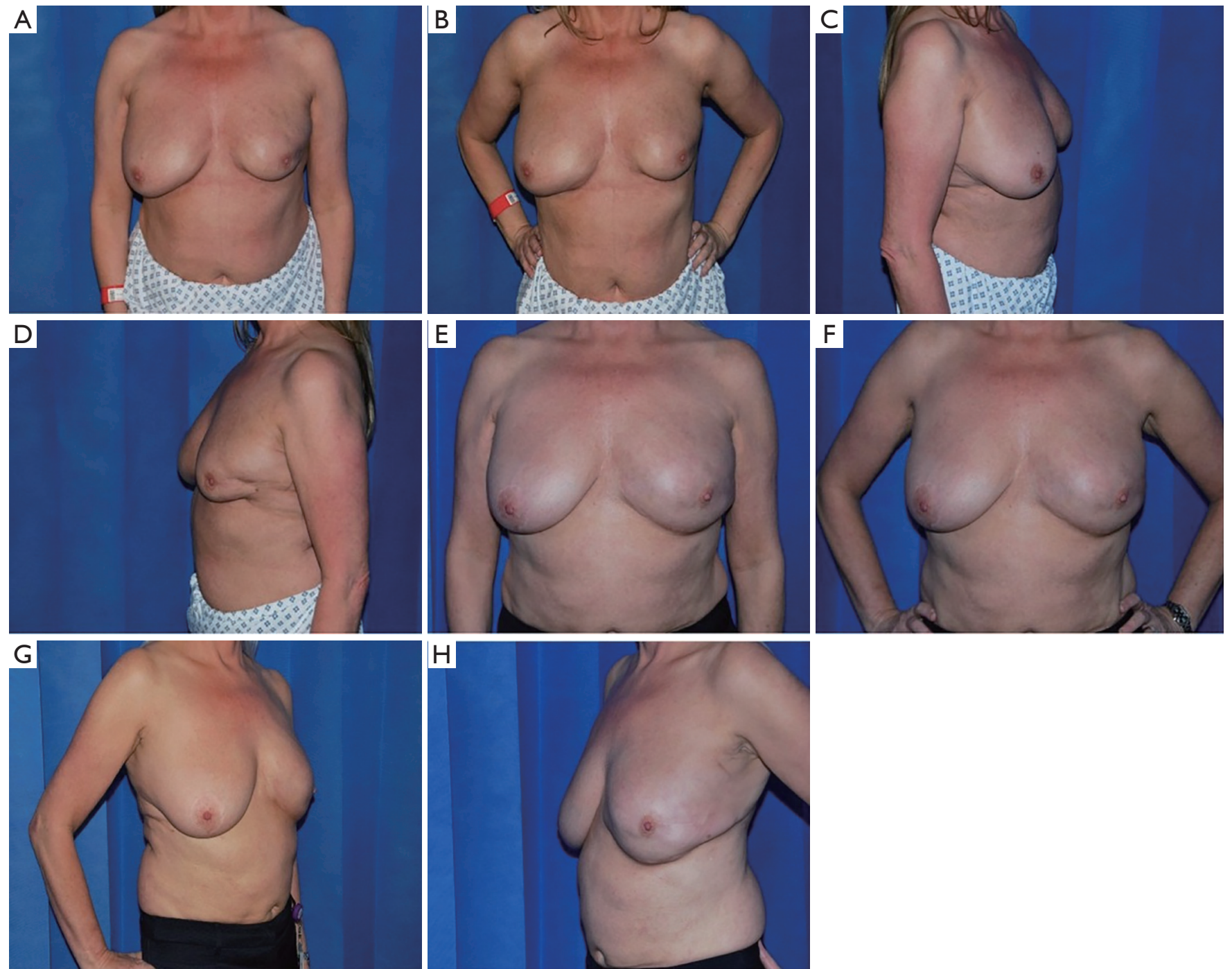

Figure 6 Pre and post-operative photographs for management of partial breast defect with pedicled local flap. (A,B,C,D) Preoperative photographs of a 55 years old lady who developed left breast lower outer quadrant defect after being treated with lumpectomy for a breast cancer (top row); (E,F,G,H) postoperative photographs of delayed reconstruction of the breast defect with MSLD flap. MSLD, muscle sparing latissimus dorsi muscle flap.

limiting the width of the muscle cuff, the transverse branch of the thoracodorsal artery and the main thoracodorsal nerve may be left in situ. However, we had to ligate the transverse branch to add more length to the TD pedicle in a few patients.

Since the introduction of MSLD flaps for total or partial breast reconstruction in mastectomy patients, using a partial strip of the LD muscle with the flap that contains a descending branch of the thoracodorsal vessels, donorsite morbidity has decreased significantly (16). In all our cases, the donor site scar was aesthetically placed in the bra strap line. Moreover, we have found that creating a tunnel from the donor site to the breast approximately at 4 O'clock position to inset the flap, allows preservation of the natural lateral breast borders with no disruption of the axillary silhouette. The likelihood of seroma formation with these flaps is very low when compared with full-width latissimus dorsi flap harvest (17). To prevent postoperative seroma formation, skin-flap fixation to the underlying structures with absorbable suture (quilting) and the use of fibrin 

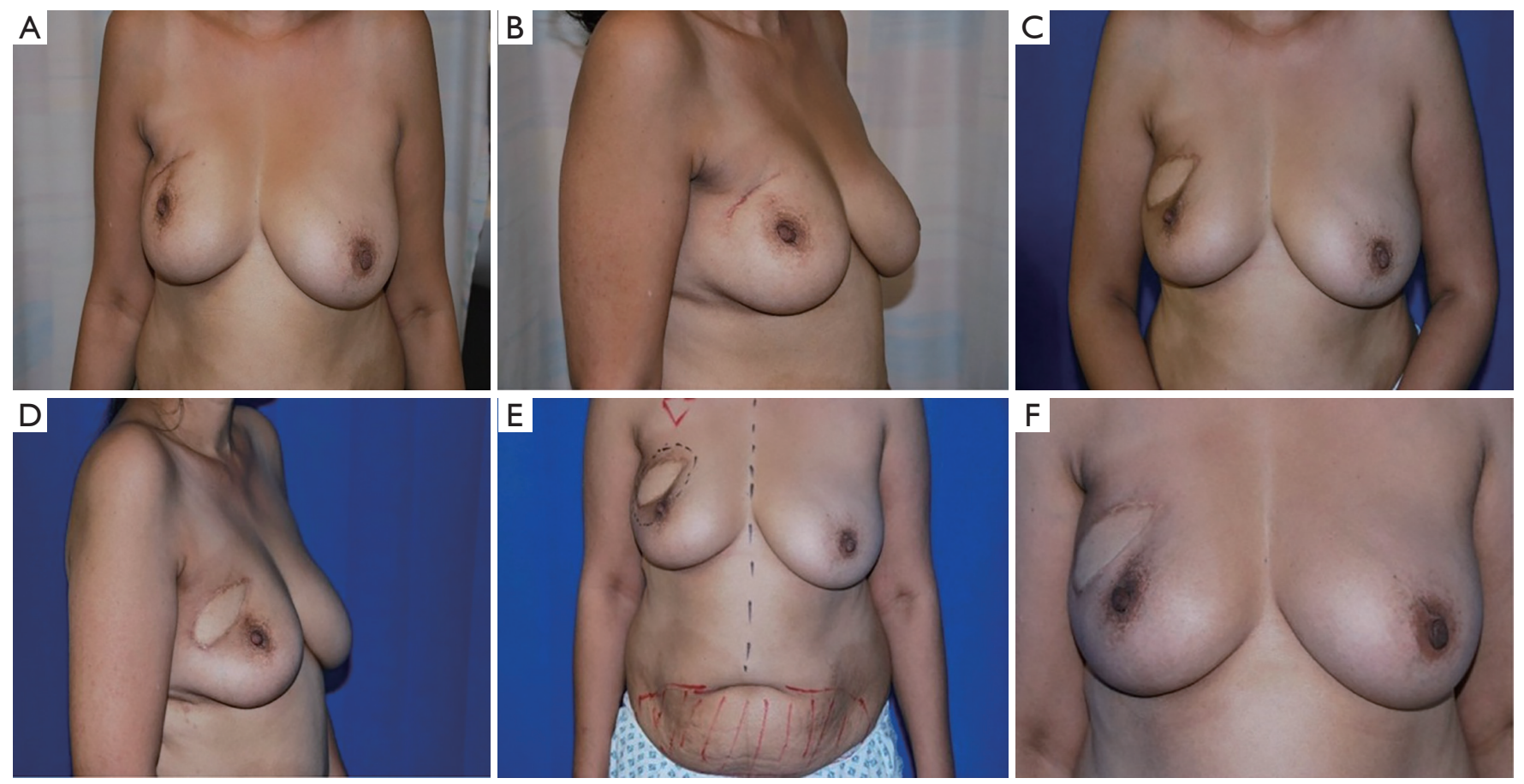

Figure 7 Role of lipofilling in enhancing cosmetic outcome and symmetry after partial breast defect reconstruction. (A,B) Preoperative photographs of a 43 years old lady who developed right breast upper outer quadrant defect after being treated with lumpectomy for a breast cancer; (C,D) postoperative photographs of delayed reconstruction of the breast defect with TDAP flap; (E) a photograph showing markings for fat grafting of the right breast as a second stage to maximize the aesthetic outcome; (F) a postoperative photo showing significant improvement in symmetry between both breasts after fat grafting. TDAP, thoraco-dorsal artery perforator flap.

sealant glue is recommended (18). In our study, despite not using this technique, we had no patients with postoperative donor site seroma. Also, we didn't experience any differences in donor site morbidity between MSLD and TDAP flaps. On one hand, this is due to less undermining and dissection which characterizes this flap procedure and on the other hand due to suturing the remaining edges of the remaining muscle with continuous suture after harvesting the flap which is a characteristic feature of our technique. We believe this step reduces any possible oozing from the muscle edges which consequently contributes to less incidence of post-operative donor site seroma. Although in this cohort we haven't formally evaluated or assessed the difference in strength or range of motion between operated and non-operated side as it is beyond the remit of this study, there was no reported cases of shoulder muscle power deficit during the clinical follow-up or in the questionnaire survey.

Interestingly, we believe that another important outcome of our study is that it demonstrates that pedicled perforator flaps can be used as a conjunct tool with other reconstructive tools in reconstruction of relatively bigger partial breast defects or when the patients' desire is for a bigger sized breast. Due to compromised blood supply after radiotherapy, reconstructing irradiated breast with lipofilling or breast prosthesis usually results in suboptimal aesthetic outcomes such as fat necrosis and capsular contracture. Pedicled perforator flaps can provide a solution for this issue by providing a healthy platform in case of fat grafting and robust coverage for breast implants $(19,20)$. It is reported that small deficit in volume after pedicled perforator flap breast reconstruction could be easily compensated for by autologous fat grafting (21). Similarly, eight of our patients underwent autologous fat grafting to supplement the volume in a second stage procedure with satisfactory aesthetic outcomes.

One of the strengths of our study is the proper preoperative preparation as well as the intra-operative and post-operative care. For instance, all patients had a preoperative mammogram and whole-body CT scan to ensure 
oncological safety by excluding the presence of new breast lesions or metastasis elsewhere in the body. Also, during patient selection, we were very determined to choose patients who have a reasonable body mass index (BMI) to minimize any obesity related complications as deep venous thrombosis and wound healing problems. Intra-operatively, all patients received intercostal and thoracodorsal nerve block to minimize the post-operative pain. As a result, less use of pain-killers, early ambulation and early discharge were achieved. Additionally, as part of the enhanced recovery program to reduce hospital stay, the physiotherapy team were involved in patients care from day 1, helping them to start mobilizing as soon as possible and providing them with a special physiotherapy plan to avoid any postoperative shoulder functional problems. Other strengths of our study are the reasonable follow-up period, assessment of patient satisfaction using a patient questionnaire survey, the reasonable satisfactory outcomes we achieved and the very low complication rate that could give a prove of how reliable this reconstructive tool in reconstruction of partial breast defects. However, there are a few limitations as the small number of cases, no randomized control trial and no detailed analysis of functional deficit on the operated side. Further study would be necessary to quantify the differences in operative times between pedicled perforator flaps and other autologous breast reconstructive procedures. Also, whether specific patient or operative factors are associated with different complications, as outlined by previous authors. Finally, future investigation to compare the aesthetic and functional outcomes of these techniques with other partial breast reconstructive procedures as lipofilling, breast prothesis and free flaps on a larger scale of patients.

\section{Conclusions}

Breast conservative surgery (BCS) can lead to unacceptable aesthetic outcomes. Each defect must be assessed on individual basis to determine the extent of volume and skin deficiency. The availability of a range of reliable techniques including thoracodorsal/intercostal artery perforator flap (TAP/ICAP) and muscle sparing latissimus dorsi flap (MSLD) allow optimum results to be achieved. Based on our experience, we believe that these flaps can be considered as "workhorse" flaps for partial breast defect reconstruction, as we found excellent aesthetic and functional results after using them in correcting post BCS deformities.

\section{Acknowledgments}

The authors acknowledge the efforts and expert input of the Medical Illustration Team at University Hospitals Birmingham NHS Foundation Trust.

\section{Footnote}

Conflicts of Interest: The authors have no conflicts of interest to declare.

Ethical Statement: The authors are accountable for all aspects of the work in ensuring that questions related to the accuracy or integrity of any part of the work are appropriately investigated and resolved. The study was approved by the Audit and Research Department in University Hospitals Birmingham and written informed consent was obtained from all patients.

\section{References}

1. Fisher B, Anderson S, Bryant J, et al. Twenty-year followup of a randomized trial comparing total mastectomy, lumpectomy, and lumpectomy plus irradiation for the treatment of invasive breast cancer. $\mathrm{N}$ Engl J Med 2002;347:1233-41.

2. Clough KB, Lewis JS, Couturaud B, et al. Oncoplastic techniques allow extensive resections for breast-conserving therapy of breast carcinomas. Ann Surg 2003;237:26.

3. Losken A, Dugal CS, Styblo TM, et al. A meta-analysis comparing breast conservation therapy alone to the oncoplastic technique. Ann Plast Surg 2014;72:145-9.

4. Hamdi M, Decorte T, Demuynck M, et al. Shoulder function after harvesting a thoracodorsal artery perforator flap. Plast Reconstr Surg 2008;122:1111-7.

5. Hamdi M, Van Landuyt K, Van Hedent E, et al. Advances in autogenous breast reconstruction: the role of preoperative perforator mapping. Ann Plast Surg 2007;58:18-26.

6. Schaverien M, Wong C, Bailey S, et al. Thoracodorsal artery perforator flap and Latissimus dorsi myocutaneous flap-anatomical study of the constant skin paddle perforator locations. J Plast Reconstr Aesthet Surg 2010;63:2123-7.

7. Youssif S, Hassan Y, Tohamy A, et al. Demonstration of MSLD in partial breast reconstruction. Asvide 2019;6:308. 
Available online: http://www.asvide.com/watch/32992

8. Saint-Cyr M, Nagarkar P, Schaverien M, et al. The pedicled descending branch muscle-sparing latissimus dorsi flap for breast reconstruction. Plast Reconstr Surg 2009;123:13-24

9. Lee KT, Lim SY, Pyun JK, et al. Improvement of upper extremity lymphedema after delayed breast reconstruction with an extended latissimus dorsi myocutaneous flap. Arch Plast Surg 2012;39:154.

10. Angrigiani C, Grilli D, Siebert J. Latissimus dorsi musculocutaneous flap without muscle. Plast Reconstr Surg 1995;96:1608-14.

11. Hamdi M, Van Landuyt K, Monstrey S, et al. Pedicled perforator flaps in breast reconstruction: a new concept. $\mathrm{Br}$ J Plast Surg 2004;57:531-9.

12. Van Landuyt K, Hamdi M, Blondeel P, et al. Autologous breast augmentation by pedicled perforator flaps. Ann Plast Surg 2004;53:322-7.

13. Kronowitz SJ, Kuerer HM, Buchholz TA, et al. A management algorithm and practical oncoplastic surgical techniques for repairing partial mastectomy defects. Plast Reconstr Surg 2008;122:1631-47.

14. Tran NV, Chang DW, Gupta A, et al. Comparison of immediate and delayed free TRAM flap breast reconstruction in patients receiving postmastectomy radiation therapy. Plast Reconstr Surg 2001;108:78-82.

15. Huizum MA, Hage JJ, Oldenburg HA, et al. Internal

Cite this article as: Youssif S, Hassan Y, Tohamy A, Eid S, Ashour T, Malahias M, Khalil H. Pedicled local flaps: a reliable reconstructive tool for partial breast defects. Gland Surg 2019;8(5):527-536. doi: 10.21037/gs.2019.09.06
Mammary Artery Perforator Flap for Immediate Volume Replacement Following Wide Local Excision of Breast Cancer. Arch Plast Surg 2017;44:502-8.

16. Brackley PT, Mishra A, Sigaroudina M, et al. Modified muscle sparing latissimus dorsi with implant for total breast reconstruction-extending the boundaries. J Plast Reconstr Aesthet Surg 2010;63:1495-502.

17. Tomita K, Yano K, Masuoka T, et al. Postoperative seroma formation in breast reconstruction with latissimus dorsi flaps: a retrospective study of 174 consecutive cases. Ann Plast Surg 2007;59:149-51.

18. Jeon BJ, Lee TS, Lim SY, et al. Risk factors for donor-site seroma formation after immediate breast reconstruction with the extended latissimus dorsi flap: a statistical analysis of 120 consecutive cases. Ann Plast Surg 2012;69:145-7.

19. Levine JL, Soueid NE, Allen RJ. Algorithm for autologous breast reconstruction for partial mastectomy defects. Plast Reconstr Surg 2005;116:762-7.

20. de Blacam C, Momoh AO, Colakoglu S, et al. Evaluation of clinical outcomes and aesthetic results after autologous fat grafting for contour deformities of the reconstructed breast. Plast Reconstr Surg 2011;128:411e-8e.

21. Kim H, Wiraatmadja ES, Lim SY, et al. Comparison of morbidity of donor site following pedicled muscle-sparing latissimus dorsi flap versus extended latissimus dorsi flap breast reconstruction. J Plast Reconstr Aesthet Surg 2013;66:640-6. 